\title{
Level of repair analysis and minimum cost
} homomorphisms of graphs

Gutin, Gregory and Rafiey, Arash and Yeo, Anders and Tso, Michael

2002

MIMS EPrint: 2006.349

Manchester Institute for Mathematical Sciences

School of Mathematics

The University of Manchester

\footnotetext{
Reports available from:

http://eprints.maths.manchester.ac.uk/

And by contacting: The MIMS Secretary

School of Mathematics

The University of Manchester

Manchester, M13 9PL, UK
} 


\title{
Communication
}

\section{Level of repair analysis and minimum cost homomorphisms of graphs}

\author{
Gregory Gutina, Arash Rafiey ${ }^{\mathrm{a}}$, Anders Yeo ${ }^{\mathrm{a}}$, Michael Tso ${ }^{\mathrm{b}}$ \\ ${ }^{a}$ Department of Computer Science, Royal Holloway University of London, Egham, Surrey TW20 OEX, UK \\ ${ }^{\mathrm{b}}$ School of Mathematics, University of Manchester, P.O. Box 88, Manchester M60 1QD,UK
}

Received 9 February 2005; received in revised form 1 May 2005; accepted 13 June 2005

Available online 29 November 2005

Communicated by C. McDiarmid

This paper is dedicated to the memory of Lillian Barros

\begin{abstract}
Level of repair analysis (LORA) is a prescribed procedure for defense logistics support planning. For a complex engineering system containing perhaps thousands of assemblies, sub-assemblies, components, etc. organized into several levels of indenture and with a number of possible repair decisions, LORA seeks to determine an optimal provision of repair and maintenance facilities to minimize overall life-cycle costs. For a LORA problem with two levels of indenture with three possible repair decisions, which is of interest in UK and US military and which we call LORA-BR, Barros [The optimisation of repair decisions using life-cycle cost parameters. IMA J. Management Math. 9 (1998) 403-413] and Barros and Riley [A combinatorial approach to level of repair analysis, European J. Oper. Res. 129 (2001) 242-251] developed certain branch-and-bound heuristics. The surprising result of this paper is that LORA-BR is, in fact, polynomial-time solvable. To obtain this result, we formulate the general LORA problem as an optimization homomorphism problem on bipartite graphs, and reduce a generalization of LORA-BR, LORA-M, to the maximum weight independent set problem on a bipartite graph. We prove that the general LORA problem is NP-hard by using an important result on list homomorphisms of graphs. We introduce the minimum cost graph homomorphism problem, provide partial results and pose an open problem. Finally, we show that our result for LORA-BR can be applied to prove that an extension of the maximum weight independent set problem on bipartite graphs is polynomial time solvable.
\end{abstract}

(c) 2005 Elsevier B.V. All rights reserved.

Keywords: Computational logistics; Level of repair analysis; Independent sets in graphs; Homomorphisms of graphs

\section{Introduction}

Level of repair analysis (LORA) is a prescribed procedure for defense logistics support planning (see e.g. [10] and the website of the UK MoD Acquisition Management System at www.ams.mod.uk/ams). For a complex engineering system containing perhaps thousands of assemblies, sub-assemblies, components etc. organized into $\ell \geqslant 2$ levels of indenture and with $r \geqslant 2$ possible repair decisions, LORA seeks to determine an optimal provision of repair and maintenance facilities to minimize overall life-cycle costs.

E-mail addresses: gutin@cs.rhul.ac.uk(G.Gutin), arash@cs.rhul.ac.uk (A.Rafiey),anders@cs.rhul.ac.uk(A. Yeo), mike.tso@manchester.ac.uk (M. Tso). 
Barros [4] and Barros and Riley [6] provide a generic integer programming formulation of the LORA optimization problem for systems with $\ell$ levels of indenture and $r$ possible repair decisions (including the non-repair option). A special case with $\ell=2$ and $r=3$, which we call LORA-BR, is of particular importance because it corresponds to recommendations in certain UK and US military standard handbooks, see [6]. In French military standards, $\ell=2$ and $r=5$. Notice that the actual research of Barros and Riley was only for LORA-BR [5] for which the corresponding software have been developed.

While Barros [4] solves LORA-BR using a general purpose IP solver, Barros and Riley [6] outline a specialized branch-and-bound heuristic, which appears to be more efficient in computational experiments. Their heuristic is based on a relaxation of LORA-BR into a pair of uncapacitated facility location (UFLP) problems [9,14]. A branch-and-bound procedure then employs local search heuristics to satisfy additional side constraints ensuring consistency between repair decisions for pairs of items nested on adjacent indenture levels. Since UFLP is $N P$-hard $[9,13,14]$, it could be expected that LORA-BR would also be intractable. However, the surprising result of this paper is that LORA-BR is polynomially solvable and this is achieved by reducing its generalization, LORA-M (defined in Section 3), to the maximum weight independent set problem on a bipartite graph.

As it was pointed out above, the case of two levels of indenture is of particular interest (e.g., in UK, USA and French military). For clarity of exposition, in the rest of this paper apart from Section 4, we restrict ourselves to two levels of indenture, $\ell=2$, but our approach can be extended to arbitrary $\ell$ as demonstrated in Section 4 .

We will use the notion of a homomorphism of graphs that generalizes the notion of coloring (see e.g. [16]). For a pair of graphs $H=(V(H), E(H))$ and $B=(V(B), E(B))$, a mapping $k: V(B) \rightarrow V(H)$ such that if $x y \in E(B)$ then $k(x) k(y) \in E(H)$ is called a homomorphism of $B$ to $H$. To study the LORA problem, we show how to formulate it as a problem of finding a homomorphism of minimum cost belonging to a certain class of homomorphisms of a bipartite graph to a fixed bipartite graph. This allows us to use a non-trivial result on the list $H$-homomorphism problem from [11] to easily show that the general LORA problem with $\ell=2$ is $N P$-hard. We also prove that LORA-M is polynomial time solvable.

The formulation of the LORA problem in terms of special homomorphisms leads us to the introduction of the minimum cost $H$-homomorphism problem (MCHP): for a fixed graph $H$ and an input graph $G$ given together with costs $c_{z}(u)$, the cost of mapping a vertex $u \in V(G)$ to $z \in V(H)$, verify whether there is a homomorphism of $G$ to $H$, and if one exists, find such a homomorphism $k$ that minimizes $\sum_{u \in V(G)} c_{k(u)}(u)$. MCHP extends the well-studied list $H$-homomorphism problem [16]. We use our results for the LORA problem to obtain the corresponding results for MCHP. In particular, we show that if $H$ is a bipartite graph with the complement being an interval graph, then MCHP is polynomial time solvable. In contrast, if $H$ is not bipartite with the complement being a circular arc graph, then MCHP is $N P$-hard.

We also use our results to show that the bipartite case of the critical independent set problem (defined in Section 6), which generalizes the maximum weight independent set problem, is polynomial time solvable.

In this paper, all graphs are finite, undirected, and simple (i.e., without loops or multiple edges). For standard graphtheoretical terminology and notation, see e.g. $[3,18]$. For terminology and results on homomorphisms, see Hell and Nesetril [16].

The rest of the paper is organized as follows. In Section 2, we provide formulations of LORA-BR and the general LORA problem with $\ell=2$ in terms of graph homomorphisms. We prove that the general LORA problem with $\ell=2$ is $N P$-hard. In Section 3, we show how to solve a generalization of LORA-BR, LORA-M with $\ell=2$, in polynomial time. In Section 4, we extend the general LORA problem with $\ell=2$ to the general LORA problem with arbitrary $\ell \geqslant 2$ as well extend the main result of Section 3. In Section 5, we introduce the minimum cost $H$-homomorphism problem and show that the results of Sections 2 and 3 can be easily extended to it. In the end of the section, we pose an open problem. Finally, in Section 6 we apply a result from Section 3 to solve the bipartite case of the critical independent set problem in polynomial time.

\section{LORA-BR and general LORA with $\ell=2$}

Consider first a special case of LORA with $\ell=2$ and $r=3$ following Barros [4] and Barros and Riley [6] (we will call this special case LORA-BR). We refer to the first level of indenture in LORA-BR as subsystems $s \in S$ and the second level of indenture as modules $m \in M$. The distribution of modules in subsystems can be given by a bipartite 
graph $G=\left(V_{1}, V_{2} ; E\right)$ with partite sets $V_{1}=S$ and $V_{2}=M$. For arbitrary $s \in V_{1}$ and $m \in V_{2}, s m \in E$ if and only if module $m$ is in subsystem $s$. We consider $G$ to be an arbitrary bipartite graph and denote its vertex set $V\left(V=V_{1} \cup V_{2}\right)$.

There are $r=3$ available repair decisions for each level of indenture: "discard", "local repair" and "central repair", labeled, respectively $D, L, C$ (subsystems) and $d, l, c$ (modules). To be able to use a decision $z \in\{D, L, C, d, l, c\}$, we have to pay a fixed $\operatorname{cost} c_{z}$. Assume also known additive costs (over a system life-cycle) $c_{z}(u)$ of prescribing repair decision $z$ for subsystem or module $u$.

We wish to minimize the total cost of choosing a subset of the six repair decisions and assigning available repair options to the subsystems and modules subject to the following constraints:

If a module $m$ occurs in subsystem $s$ (i.e., $s m \in E$ ) we impose the following logical restrictions on the repair decisions for the pair $(s, m)$ motivated through practical considerations:

$$
R_{1}: D_{s} \Rightarrow d_{m}, R_{2}: l_{m} \Rightarrow L_{s},
$$

where $D_{s}, d_{m}$ denote the decisions to discard subsystem $s$, module $m$, respectively, etc. Notice that even though module $m$ may be common to several subsystems we are required to prescribe a unique repair decision for that module.

$R_{1}$ has the interpretation that a decision to discard subsystem $s$ necessarily entails discarding all enclosed modules. $R_{2}$ is a consequence of $R_{1}$ and a policy of "no backshipment" which rules out the local repair option for any module enclosed in a subsystem which is sent for central repair [6].

Let $F_{B R}=\left(Z_{1}, Z_{2} ; T\right)$ be a bipartite graph with partite sets $Z_{1}=\{D, C, L\}$ (subsystem repair options) and $Z_{2}=$ $\{d, c, l\}$ (module repair options) and with edges $T=\{D d, C d, C c, L d, L c, L l\}$. Let $Z=Z_{1} \cup Z_{2}$. Observe that any homomorphism $k$ of $G$ to $F_{B R}$ such that $k\left(V_{1}\right) \subseteq Z_{1}$ and $k\left(V_{2}\right) \subseteq Z_{2}$ satisfies the rules $R_{1}$ and $R_{2}$. Indeed, let $u \in V_{1}$, $v \in V_{2}, u v \in E$. If $k(u)=D$ then $k(v)=d$, and if $k(v)=l$ then $k(u)=L$.

Let $L_{i} \subseteq Z_{i}, i=1,2$. We call a homomorphism $k$ of $G$ to $F_{B R}$ an $\left(L_{1}, L_{2}\right)$-homomorphism of $G$ to $F_{B R}$ if $k(u) \in L_{i}$ for each $u \in V_{i}, i=1,2$. Now LORA-BR can be formulated as the following graph-theoretical problem: we are given a bipartite graph $G=\left(V_{1}, V_{2} ; E\right), V=V_{1} \cup V_{2}$, and we consider homomorphisms $k$ of $G$ to $F_{B R}$. (If no homomorphisms of $G$ to $F_{B R}$, then the problem has no feasible solution.) Mapping of $u \in V$ to $z \in Z$ (i.e., $k(u)=z$ ) incurs a real cost $c_{z}(u)$. The use of a vertex $z \in Z$ in a homomorphism $k$ (i.e., $k^{-1}(z) \neq \emptyset$ ) incurs a real cost $c_{z}$. We wish to choose subsets $L_{i} \subseteq Z_{i}, i=1,2$, and find an $\left(L_{1}, L_{2}\right)$-homomorphism $k$ of $G$ to $F_{B R}$ that minimize

$$
\sum_{u \in V} c_{k(u)}(u)+\sum_{z \in L_{1} \cup L_{2}} c_{z}
$$

We call the expression in (1) the cost of $k$.

The graph-theoretical formulation of LORA-BR can be naturally extended as follows: The above problem with $F_{B R}$ replaced by an arbitrary fixed bipartite graph $F=\left(Z_{1}, Z_{2} ; T\right)$ is called the general LORA problem with $\ell=2$. Let $Z=Z_{1} \cup Z_{2}$. Notice that the general LORA problem with $\ell=2$ extends the generic formulation of the LORA problem with $\ell=2$ given in [6]. The formulation of the general LORA problem (with arbitrary $\ell$ ) provided in Section 4 extends the generic formulation of the LORA problem (with arbitrary $\ell$ ) given in [6].

To prove that the general LORA problem with $\ell=2$ is $N P$-hard, we will use an important result on the list $H$ homomorphism problem defined below. Suppose that we are given a pair of graphs $H$ and $B$ and a list $\Lambda(v) \subseteq V(H)$ for each $v \in V(B)$. A homomorphism $f: V(B) \rightarrow V(H)$ such that $f(v) \in \Lambda(v)$ for each $v \in V(B)$ is called a $\Lambda$-homomorphism. For a fixed $H$, the list $H$-homomorphism problem asks whether there exists a $\Lambda$-homomorphism $f$ of $B$ to $H$ for an input graph $B$ with lists $\Lambda$.

A graph $P=(V(P), E(P))$ is a circular arc graph if there is a family of arcs $A_{v}, v \in V(P)$, on a fixed circle, such that $x y \in E(P)$ if and only if $A_{x}$ and $A_{y}$ intersect. Feder et al. [11] obtained the following important result:

Theorem 1. If $H$ is a bipartite graph with the complement being a circular arc graph, then the list H-homomorphism problem is polynomial time solvable. Otherwise, the problem is NP-complete.

Observe that, if $H$ is bipartite, we may restrict inputs $B$ of the list $H$-homomorphism problem to bipartite graphs since there is no homomorphism of a non-bipartite graph to $H$. Brightwell [7] found the first proof that the general LORA problem with $\ell=2$ is $N P$-hard. Since his proof does not use Theorem 1, our proof turns out to be shorter and it covers much wider family of graphs than that of Brightwell. 
Theorem 2. The general LORA problem with $\ell=2$ and with each $c_{z}=0$, and each $\operatorname{cost} c_{z}(u)$ in $\{0,1\}$ is NP-hard provided the complement of $F$ is not a circular arc graph.

Proof. Let $F$ be a bipartite graph and assume that the complement of $F$ is not a circular arc graph (see Theorem 1). Let a bipartite graph $G$ and lists $\Lambda$ be an input of the list $F$-homomorphism problem. Define costs $c_{z}(u)$ for each $z \in V(F)$ and $u \in V(G)$ as follows: $c_{z}(u)=0$ if $z \in \Lambda(u)$ and $c_{z}(u)=1$, otherwise. We put $c_{z}=0$ for each $z \in V(F)$. In other words, the use of each vertex $z \in V(F)$ in homomorphisms of $G$ to $H$ is free. In this case, in the general LORA problem with $\ell=2$, we can always put $L_{1} \cup L_{2}=V(F)$.

Let $G_{1}, G_{2}, \ldots, G_{g}$ be components of $G$ and let $F_{1}, F_{2}, \ldots, F_{f}$ be components of $F$. Let $Z_{1}^{j}$, $Z_{2}^{j}$ be partite sets of $F_{j}$ for every $j=1,2, \ldots, f$. Observe that there exists a $\Lambda$-homomorphism of $G$ to $F$ if and only if for each $i=1,2, \ldots, g$ there is a $j(i) \in\{1,2, \ldots, f\}$ such that there exists a $\Lambda$-homomorphism of $G_{i}$ to $F_{j(i)}$. However, there is a $\Lambda$-homomorphism of $G_{i}$ to $F_{j(i)}$ if and only if the minimum cost of either a $\left(Z_{1}^{j(i)}, Z_{2}^{j(i)}\right)$-homomorphism of $G_{i}$ to $F_{j(i)}$ or a $\left(Z_{2}^{j(i)}, Z_{1}^{j(i)}\right)$-homomorphism of $G_{i}$ to $F_{j(i)}$ is equal to 0 (with the costs defined above). Thus, we have a polynomial time Turing-reduction [13] from the $N P$-complete list $H$-homomorphism problem to the general LORA problem with $\ell=2$. Hence, by the definition of the $N P$-hardness (see [13, Section 5.1]), the general LORA problem with $\ell=2$ is $N P$-hard.

It is well-known [15] (see also [16]) that for a fixed graph $H$, the problem to verify whether there exists a homomorphism of an input graph $G$ into $H$ is $N P$-complete if $H$ is non-bipartite and polynomial time solvable if $H$ is bipartite. Thus, the obvious extension of the general LORA problem to non-bipartite graphs $F$ is $N P$-hard.

\section{LORA-M with $\ell=2$}

Let $B=\left(W_{1}, W_{2} ; E\right)$ be a bipartite graph. For a vertex $z \in W_{1} \cup W_{2}$, let $N(z)$ be the set of vertices adjacent to $z$. Orderings $x_{1}, x_{2}, \ldots, x_{\left|W_{1}\right|}$ and $y_{1}, y_{2}, \ldots, y_{\left|W_{2}\right|}$ of vertices of $W_{1}$ and $W_{2}$, respectively, are called monotone if $N\left(x_{i}\right) \subseteq N\left(x_{i+1}\right)$ and $N\left(y_{j}\right) \subseteq N\left(y_{j+1}\right)$ for each $i=1,2, \ldots,\left|W_{1}\right|-1$ and $j=1,2, \ldots,\left|W_{2}\right|-1$. A bipartite graph $B$ is called monotone if it has monotone orderings of its partite sets. Observe that if $x_{1}, x_{2}, \ldots, x_{\left|W_{1}\right|}$ and $y_{1}, y_{2}, \ldots, y_{\left|W_{2}\right|}$ are monotone orderings, then $x_{p} y_{q} \in E$ implies that $x_{s} y_{t} \in E$ for each $s \geqslant p$ and $t \geqslant q$.

Notice that the bipartite graph $F_{B R}$ corresponding to the rules $R_{1}$ and $R_{2}$ of LORA-BR is monotone (consider orderings $D, C, L$ and $l, c, d$ ), so are the bipartite graphs corresponding to $R_{1}$ and $R_{2}$ separately (there might be a situation when one of the rules is not used). Interestingly, monotone bipartite graphs form a family of so-called convex bipartite graphs; several families of convex bipartite graphs have been found useful in various applications, see [3].

Let $B=\left(W_{1}, W_{2} ; E\right)$ be a bipartite graph, let $n=\left|W_{1}\right|+\left|W_{2}\right|$ and let $m=|E|$. One can test whether $B$ is monotone in time $\mathrm{O}(m+n)$ as follows. Order vertices of $W_{1}$ and $W_{2}$ separately according to their degrees $\operatorname{deg}(z), x_{1}, x_{2}, \ldots, x_{\left|W_{1}\right|}$ and $y_{1}, y_{2}, \ldots, y_{\left|W_{2}\right|}$, such that $\operatorname{deg}\left(x_{i}\right) \leqslant \operatorname{deg}\left(x_{i+1}\right)$ and $\operatorname{deg}\left(y_{j}\right) \leqslant \operatorname{deg}\left(y_{j+1}\right)$ for each $i=1,2, \ldots,\left|W_{1}\right|-1$ and $j=1,2, \ldots,\left|W_{2}\right|-1$. Observe that $B$ is monotone if and only if these orderings are monotone. We can use counting sort (see [8, Chapter 9]) to get the orderings according to degrees in time $\mathrm{O}(n)$. The remaining computations can be carried out in time $\mathrm{O}(\mathrm{m})$.

The general LORA problem restricted to fixed monotone bipartite graphs $F=\left(Z_{1}, Z_{2} ; T\right)$ is called LORA-M. We assume that we have monotone orderings $x_{1}, x_{2}, \ldots, x_{\left|Z_{1}\right|}$ and $y_{1}, y_{2}, \ldots, y_{\left|Z_{2}\right|}$ of $Z_{1}$ and $Z_{2}$, respectively.

We reduce LORA-M to the maximal weight independent set problem on bipartite graphs. Recall that a vertex set $I$ of a graph is independent if there is no edge between vertices of $I$.

In the next theorem, we will consider a bipartite graph $B$ with partite sets $W_{1}, W_{2}$ and non-negative vertex weights $p(u), u \in V(B)$, and the following $(s, t)$-network $\mathscr{N}(B)$ : add new vertices $s$ and $t$ to $B$, append all arcs $s u$ of capacity $p(u), v t$ of capacity $p(v)$ for all $u \in W_{1}$ and $v \in W_{2}$, and orient every edge $x y$ of $B$, where $x \in W_{1}$, from $x$ to $y$ (these arcs are of capacity $\infty$ ). For results on flows and cuts in networks see [8].

Theorem 3. If $(S, T)$ is a minimum cut in $\mathscr{N}(B), s \in S$, then $\left(S \cap W_{1}\right) \cup\left(T \cap W_{2}\right)$ is a maximum weight independent set in B. One can find a maximum weight independent set in $B$ in time $\mathrm{O}\left(n_{1}^{2} \sqrt{m}+n_{1} m\right)$, where $n_{1}=\left|W_{1}\right|$ and $m=|E(B)|$.

The structural part of Theorem 3 is well-known, cf. Frahling and Faigle [12] (a similar result is described in [17]). 
The complexity claim follows from the fact that one can find a minimum cut in $\mathscr{N}(B)$ in time $\mathrm{O}\left(n_{1}^{2} \sqrt{m}+n_{1} m\right)$ by first finding a maximum flow by the bipartite preflow-push algorithm of Ahuja et al. [2] and then finding a minimum cut (e.g., by finding vertices reachable from $s$ in the residual network using depth-first search).

Let us return to LORA-M and formulate it as a maximization problem. Choose sets $L_{i} \subseteq Z_{i}, i=1$, 2. Let $u \in V_{i}$ and set lists $\Lambda(u)=L_{i}, i=1,2$. Recall that $x_{1}, x_{2}, \ldots, x_{\left|Z_{1}\right|}$ and $y_{1}, y_{2}, \ldots, y_{\left|Z_{2}\right|}$ are monotone orderings of $Z_{1}$ and $Z_{2}$. Assume that $u \in V_{1}, x_{p}, x_{q} \in \Lambda(u), p<q$ and $c_{x_{p}}(u)>c_{x_{q}}(u)$. Observe that since $c_{x_{p}}(u)>c_{x_{q}}(u)$ and $F$ is monotone, an optimal $\left(L_{1}, L_{2}\right)$-homomorphism $k$ will not map $u$ to $x_{p}$. Thus, we may reduce the list $\Lambda(u)$ of possible images of $u$ by deleting $x_{p}$. Certainly, we may reduce all $\Lambda(v), v \in V_{1}$, such that if $x_{r}, x_{s} \in \Lambda(v)$ and $r<s$, then $c_{x_{r}}(v) \leqslant c_{x_{s}}(v)$. We call such a list $\Lambda(v)$ reduced. Similarly, one defines the reduced list of a vertex in $V_{2}$.

For a vertex $u \in V$, we can get the reduced list $\Lambda(u)$ in time $\mathrm{O}(1)$ by the following simple procedure (the running time is constant since $F$ is fixed). To simplify the description, assume that $u \in V_{1}$. The input is $\Lambda(u)$ : $=L_{1}=\left\{x_{p(1)}, x_{p(2)}, \ldots, x_{p(t)}\right\}, p(1)<p(2)<\cdots<p(t)$. We start from $x_{p(t)}$. We compare $c_{x_{p(t)}}(u)$ with $c_{x_{p(t-1)}}(u)$, $c_{x_{p(t-2)}}(u), \ldots$ and find the maximal $i$ such that $c_{x_{p(i)}}(u) \leqslant c_{x_{p(t)}}(u)$. We delete from $\Lambda(u)$ all $x_{p(i+1)}, x_{p(i+2)}, \ldots, x_{p(t-1)}$. We compare $c_{x_{p(i)}}(u)$ with $c_{x_{p(i-1)}}(u), c_{x_{p(i-2)}}(u), \ldots$ and continue as above. Thus, we can obtain the reduced lists $\Lambda(v)$, $v \in V$, in time $\mathrm{O}(|V|)$.

In the reminder of this section, we will use the following notation for the reduced lists: $\Lambda(u)=\left\{z_{p(1)}\right.$, $\left.z_{p(2)}, \ldots, z_{p(|\Lambda(u)|)}\right\}$, where $p(1)<p(2)<\cdots<p(|\Lambda(u)|)$ and $z=x$ if $u \in V_{1}$ and $z=y$, otherwise.

Recall that a homomorphism $k$ of $G$ to $F$ is a $\Lambda$-homomorphism if $k(u) \in \Lambda(u)$ for each $u \in V$. Observe that LORA-M is equivalent to the problem of choosing sets $L_{i} \subseteq Z_{i}, i=1,2$ and finding a $\Lambda$-homomorphism $k$ of $G$ to $F$ that minimize the cost of $k$, where $\Lambda(u)$ is the reduced list for $u \in V$.

Now we replace the costs by weights. Let $M$ be the maximum of all costs in LORA-M (i.e., $c_{z}(u)$ 's and $c_{z}$ 's). For each pair of vertices $z \in Z_{i}$ and $u \in V_{i}, i=1,2$, let $w_{z}(u)=M-c_{z}(u)$ and for each vertex $z \in Z$ let $w_{z}=M-c_{z}$. Notice that, by the definition, all the weights are non-negative. Let $k$ be a $\Lambda$-homomorphism of $G$ to $F$. The weight of $k$ is defined as

$$
\sum_{u \in V} w_{k(u)}(u)+\sum_{z \in L_{1} \cup L_{2}} w_{z} .
$$

Observe that LORA-M is equivalent to the problem of choosing sets $L_{i} \subseteq Z_{i}, i=1,2$ and finding a $\Lambda$-homomorphism $k$ of $G$ to $F$ that maximize the weight of $k$, where $\Lambda(u)$ is the reduced list for $u \in V$.

We now prove the following main result of the paper.

Theorem 4. For fixed subsets $L_{i}, i=1,2, L O R A-M$ with $\ell=2$ can be solved in time $\mathrm{O}\left(n_{1}^{2} \sqrt{m}+n_{1} m+n\right)$, where $n_{1}=\left|V_{1}\right|, n=|V|$ and $m=|E|$.

Proof. Recall that all our graphs have no loops. If $F$ is edgeless, then there is no homomorphism of $G$ to $F$. Thus, we may assume that $x_{\left|U_{1}\right|} y_{\left|U_{2}\right|} \in T$. Since $L_{i}, i=1,2$, are fixed, for simplicity, we will assume that all weights $w_{i j}=0$ in (2). Let $\Lambda(u)$ be the reduced list for each $u \in V$ (we have shown how to find these lists in time $\mathrm{O}(n)$ ).

Let $W$ be a constant larger than $\max \left\{w_{j}(u): u \in V, j \in \Lambda(u)\right\}$. Construct a new graph $H$ with $\sum_{u \in V}|\Lambda(u)|$ vertices

$$
V(H)=\left\{u_{z}: u \in V, z \in \Lambda(u)\right\} .
$$

Let an edge $u_{x} v_{y}$ be in $H$ if $u v \in E$ and $x y \notin T$. Let $u \in V$. For every $j \in\{1,2, \ldots,|\Lambda(u)|\}$, let the weight $w\left(u_{z_{p(j)}}\right)$ be equal to $w_{z_{p(j)}}(u)+W$, if $j=|\Lambda(u)|$, and equal to $w_{z_{p(j)}}(u)-w_{z_{p(j+1)}}(u)$, otherwise. Since each list $\Lambda(u)$ is reduced, the weights of the vertices of $H$ are non-negative.

Clearly, if we replace, in $G$, a vertex $u \in V$ by $|\Lambda(u)|$ independent copies such that there is an edge between a copy of $u$ and a copy of $v$ if and only if $u v \in E$, then we obtain a supergraph $G^{*}$ of $H$. Since $G$ is bipartite, so is $G^{*}$ and, thus, $H$.

Observe that, by monotonicity of $F$, if $u_{x_{p(i)}}, u_{x_{p(j)}}, v_{y_{p(f)}}, v_{y_{p(g)}}$ are vertices of $H, j \geqslant i, g \geqslant f$ and $u_{x_{p(i)}}$ $v_{y_{p(f)}} \notin E(H)$, then $u_{x_{p(j)}} v_{y_{p(g)}} \notin E(H)$ as well. We call this property of $H$ index-antimonotonicity. 
Assume that there exists a $\Lambda$-homomorphism $k$ of $G$ to $F$. Let $k(u)=z_{p\left(i_{u}\right)}$. Then the set $\left\{u_{z_{p\left(i_{u}\right)}}: u \in V\right\}$ is independent in $H$. Moreover, by index-antimonotonicity of $H$,

$$
S=\cup_{u \in V}\left\{u_{z_{p(j)}}: i_{u} \leqslant j \leqslant|\Lambda(u)|\right\}
$$

is an independent set in $H$. Observe that $S$ contains $S^{\prime}=\left\{u_{z_{p(A(u))}}: u \in V\right\}$ and the weight of $S$ is equal to that of the homomorphism plus $W \times|V|$ (we use telescopic sums).

Assume that a maximum weight independent set $S$ in $H$ contains $S^{\prime}$. Then map each $u \in V$ to $k(u)=z_{p\left(i_{u}\right)}$ such that $i_{u}=\min \left\{j: u_{z_{p(j)}} \in S\right\}$. By maximality, $S$ is of the form (3) or, due to index-antimonotonicity of $H, S$ may be extended to (3) by adding some vertices of zero weight. Observe that the weight of $S$ is equal to that of the homomorphism plus $W \times|V|$. If a maximum weight independent set $S$ in $H$ does not contain $S^{\prime}$, then $S^{\prime}$ is not an independent set in $H$ (since the weight of $S^{\prime}$ is larger than the weight of $S$ ) and, thus, there is no $\Lambda$-homomorphism of $G$ to $F$.

Thus, there is an $\Lambda$-homomorphism of $G$ to $F$ if and only if a maximum weight independent set in $H$ contains $S^{\prime}$. If there is an $\Lambda$-homomorphism of $G$ to $F$, then this homomorphism corresponds to a maximum weight independent set $S$ in $H$. It remains to observe that we may apply Theorem 3 to find a maximum weight independent set of $H$.

There are less than $a=2^{\left|Z_{1}\right|+\left|Z_{2}\right|}$ choices of non-empty $L_{1}$ and $L_{2}$. Since $F$ is fixed, $a$ is a constant. Thus, we obtain the following:

Theorem 5. LORA-M with $\ell=2$ can be solved in time $\mathrm{O}\left(n_{1}^{2} \sqrt{m}+n_{1} m+n\right)$, where $n_{1}, n$ and $m$ are defined in Theorem 4.

\section{General LORA problem and LORA-M}

Let $\ell \geqslant 2$ be a constant. An $\ell$-partition $X_{1}, X_{2}, \ldots, X_{\ell}$ of a set $X$ is a collection of subsets of $X$ such that $X_{i} \cap X_{j}=\emptyset$ for each $i \neq j$ and $X_{1} \cup X_{2} \cup \cdots \cup X_{\ell}=X$. An $\ell$-partition $X_{1}, X_{2}, \ldots, X_{\ell}$ of the vertex set $X$ of a graph $H$ is called layered if, for each edge $x y$ of $H$, there exists an index $i$ such that one vertex of $x y$ is in $X_{i}$ and the other is in $X_{i+1}$. Observe that a graph $H$ with a layered $\ell$-partition is bipartite with partite sets $\cup\left\{X_{i}: 1 \leqslant i \leqslant \ell, i \equiv 1(\bmod 2)\right\}$ and $\cup\left\{X_{i}: 1 \leqslant i \leqslant \ell, i \equiv 0(\bmod 2)\right\}$.

Let $G=(V, E)$ be a graph with a layered $\ell$-partition $V_{1}, V_{2}, \ldots, V_{\ell}$ of $V$. Let $F=(U, T)$ be a fixed graph with a layered $\ell$-partition $U_{1}, U_{2}, \ldots, U_{\ell}$ of $U$. Let $L_{i} \subseteq U_{i}, i=1,2, \ldots, \ell$. We call a homomorphism $k$ of $G$ to $F$ an $\left(L_{1}, L_{2}, \ldots, L_{\ell}\right)$-homomorphism of $G$ to $H$ if $k(u) \in L_{i}$ for each $u \in V_{i}, i=1,2, \ldots, \ell$.

We formulate the general LORA problem as follows: we are given a graph $G$ as above and we consider homomorphisms $k$ of $G$ to $F$. Mapping $u \in V$ to $z \in U$ (i.e., $k(u)=z$ ) incurs a real cost $c_{z}(u)$. The use of a vertex $z \in U$ in a homomorphism $k$ (i.e., $k^{-1}(z) \neq \emptyset$ ) incurs a real $\operatorname{cost} c_{z}$. We wish to choose subsets $L_{i} \subseteq U_{i}, i=1,2, \ldots, \ell$, and find an $\left(L_{1}, L_{2}, \ldots, L_{\ell}\right)$-homomorphism $k$ of $G$ to $F$ that minimizes

$$
\sum_{u \in V} c_{k(u)}(u)+\sum_{z \in L} c_{z}
$$

where $L=\cup_{i=1}^{\ell} L_{i}$. Notice that the graph $F$ is fixed and is not part of the input.

By Theorem 2, the general LORA problem is $N P$-hard (even the general LORA problem in which all costs $c_{z}(u)=0$ for $u \in V_{i}, i \geqslant 3$, is $N P$-hard). To define (the general) LORA-M for $\ell \geqslant 2$, let us define $\ell$-monotone graphs. Let $F=(U, T)$ be a fixed graph with a layered $\ell$-partition $U_{1}, U_{2}, \ldots, U_{\ell} ; F$ is called $\ell$-monotone if there is an ordering $z_{1}^{i}, z_{2}^{i}, \ldots, z_{\left|U_{i}\right|}^{i}$ of vertices of $U_{i}$ for each $i=1,2, \ldots, \ell$ such that the subgraph $F\left[U_{j} \cup U_{j+1}\right]$ of $F$ induced by $U_{j} \cup U_{j+1}$ is monotone with $z_{1}^{j}, z_{2}^{j}, \ldots, z_{\left|U_{j}\right|}^{j}$ and $z_{1}^{j+1}, z_{2}^{j+1}, \ldots, z_{\left|U_{j+1}\right|}^{j+1}$ being monotone orderings for each $j=1,2, \ldots, \ell-1$. LORA-M is the general LORA problem with $F$ being $\ell$-monotone. Similar to Theorem 5, one can prove the following:

Theorem 6. LORA-M with fixed $\ell \geqslant 2$ can be solved in time $\mathrm{O}\left(n_{1}^{2} \sqrt{m}+n_{1} m+n\right)$, where $n_{1}$ is the number of vertices in the smaller partite set of input graph $G, n=|V(G)|$ and $m=|E(G)|$. 


\section{Minimum cost $\boldsymbol{H}$-homomorphism problem}

This paper provides a motivation to study the following minimum cost H-homomorphism problem (MCHP): for a fixed graph $H$ and an input graph $G$ given together with costs $c_{z}(u)$, the cost of mapping a vertex $u \in V(G)$ to $z \in V(H)$, verify whether there is a homomorphism of $G$ to $H$, and if one exists, find such a homomorphism $k$ that minimizes $\sum_{u \in V(G)} c_{k(u)}(u)$.

An argument similar to that in the proof of Theorem 2 shows that MCHP problem generalizes the list $H$-homomorphism problem and that if $H$ is not bipartite with the complement being circular arc graph, then MCHP is NP-hard.

Theorem 7. If $H=\left(U_{1}, U_{2}, ; T\right)$ is a monotone bipartite graph, then MCHP can be solved in time $\mathrm{O}\left(n^{2} \sqrt{m}+n m+n\right)$, where $n$ is the number of vertices in the input graph $G$ and $m$ is the number of edges in $G$.

Proof. Let $t(n, m)=\mathrm{O}\left(n^{2} \sqrt{m}+n m+n\right)$. Since $H$ is bipartite (and loopless), if there is a homomorphism of $G$ to $H$, then $G$ is bipartite. So we may assume that $G=\left(V_{1}, V_{2} ; E\right)$ is bipartite.

Assume that $G$ and $H$ are connected. Then for each homomorphism $k$ of $G$ to $H$, we have either $k\left(V_{i}\right) \subseteq U_{i}$ or $k\left(V_{i}\right) \subseteq U_{3-i}$ for every $i=1,2$. Thus, to find an optimal homomorphism of $G$ to $H$, it suffices to compute an optimal $\left(U_{1}, U_{2}\right)$-homomorphism and optimal $\left(U_{2}, U_{1}\right)$-homomorphism and compare their costs. By Theorem 4 , the total running time for finding the two optimal homomorphisms is $t(n, m)$.

If $H$ is disconnected, then by the definition of monotonicity, $H$ consists of isolated vertices and at most one component $H^{\prime}$, which is not an isolated vertex. The case when all components of $H$ are isolated vertices is trivial, so we may assume that $H^{\prime}$ does exist.

Assume that $G$ consists of components $G_{1}, G_{2}, \ldots, G_{b}$. Observe that every homomorphism $k$ of $G$ to $H$ consists of $b$ "independent" homomorphisms $k_{i}: G_{i} \rightarrow H$. In fact, if $G_{i}$ has more than one vertex that $k_{i}$ maps $G_{i}$ into $H^{\prime}$ and, by the above, we can find an optimal homomorphism of $G_{i}$ to $H^{\prime}$ in time $t\left(n_{i}, m_{i}\right)$, where $n_{i}=\left|V\left(G_{i}\right)\right|$ and $m_{i}=\left|E\left(G_{i}\right)\right|$. If $G_{i}$ is a vertex $v, k_{i}$ may map it to any vertex of $H$ and, in an optimal $k_{i}$ it maps $G_{i}$ into $z$ with minimum $c_{z}(u), z \in U_{1} \cup U_{2}$. The running time to find such a vertex $z$ is $t(1,0)=\mathrm{O}(1)$. To complete our proof, it suffices to observe that $\sum_{i=1}^{b} t\left(n_{i}, m_{i}\right)=t(n, m)$.

The following theorem allows us to relate the $N P$-hardness and polynomial solvable cases above. Recall that a graph $P=(V(P), E(P))$ is an interval graph if there is a family of intervals $I_{v}, v \in V(P)$, of the real line, such that $x y \in E(P)$ if and only if $I_{x}$ and $I_{y}$ intersect. The clique covering number of a graph $B$ is the minimum number of complete subgraphs of $B$ covering $V(B)$.

Theorem 8. A graph $H$ is a monotone bipartite graph if and only if its complement $\bar{H}$ is an interval graph with clique covering number two.

Proof. First assume that $H$ is a monotone bipartite graph with partite sets $\left\{v_{1}, v_{2}, \ldots, v_{k}\right\}$ and $\left\{w_{1}, w_{2}, \ldots, w_{l}\right\}$. By the definition of a bipartite monotone graph we may assume that $v_{i} w_{j} \in E(H)$ implies that $v_{i^{\prime}} w_{j^{\prime}} \in E(H)$ for all $i^{\prime} \geqslant i$ and $j^{\prime} \geqslant j$. Let $m(j)$ be defined as the least index such that $v_{m(j)} w_{j} \in E(H)$. Now consider the following intervals:

$$
\begin{array}{ll}
s_{i}=[i, k+1] & \text { for all } i=1,2, \ldots, k, \\
t_{j}=\left[0, m(j)-\frac{1}{2}\right] & \text { for all } j=1,2, \ldots, l .
\end{array}
$$

Let $B$ be the interval graph obtained from the above intervals, such that $V(B)=S \cup T$, where $S=\left\{s_{1}, s_{2}, \ldots, s_{k}\right\}$ and $T=\left\{t_{1}, t_{2}, \ldots, t_{l}\right\}$ and there is an edge between two vertices if and only if the corresponding intervals intersect. Note that both $S$ and $T$ form a clique in $B$. Furthermore $s_{i} t_{j} \in E(B)$ if and only if $i<m(j)$, which happens if and only if $v_{i} w_{j} \notin E(H)$. Therefore, $B=\bar{H}$, and we have completed one direction.

So assume that $\bar{H}$ is an interval graph with clique covering number two. Let $\left[s_{i}, t_{i}\right], i=1,2, \ldots, k$, denote the intervals corresponding to one of the cliques in the clique cover of size two and let $\left[s_{i}^{\prime}, t_{i}^{\prime}\right], i=1,2, \ldots, l$, denote the intervals corresponding to the other clique in the clique cover. Let $T$ denote the minimum value of all $t_{i}$ and let $T^{\prime}$ denote the minimum value of all $t_{i}^{\prime}$. Without loss of generality we may assume that $T \leqslant T^{\prime}$. Again without loss of generality we may assume that $t_{1} \geqslant t_{2} \geqslant \cdots \geqslant t_{k}$ and $s_{1}^{\prime} \leqslant s_{2}^{\prime} \leqslant \cdots \leqslant s_{l}^{\prime}$. 
Assume that $\left[s_{i}, t_{i}\right]$ and $\left[s_{j}^{\prime}, t_{j}^{\prime}\right]$ do not intersect. Suppose that $t_{j}^{\prime}<s_{i}$, which implies that $t_{k}<s_{i}$ contradicting the fact that $\left[s_{k}, t_{k}\right]$ and $\left[s_{i}, t_{i}\right]$ intersect. Therefore, we must have $t_{i}<s_{j}^{\prime}$, which implies that $\left[s_{a}, t_{a}\right]$ and $\left[s_{b}^{\prime}, t_{b}^{\prime}\right]$ do not intersect for any $a \geqslant i$ and $b \geqslant j$. Therefore $\bar{H}$ is the complement of a monotone bipartite graph.

The last two theorems imply the following:

Theorem 9. If $H$ is a bipartite graph and its complement is an interval graph, then MCHP can be solved in time $\mathrm{O}\left(n^{2} \sqrt{m}+n m+n\right)$, where $n$ is the number of vertices in an input graph $G$ and $m$ is the number of edges in $G$.

Let $P_{5}$ be the path with five vertices. The graph $P_{5}$ is not a monotone bipartite graph, but its complement is a circular arc graph. Thus, there remains a gap between the set of graphs $H$ for which we showed that the problem is $N P$-hard and for which we proved that it is tractable. It would be interesting to close the gap. We considered some directed extension of the 2-SAT approach of [11], but they did not appear to be useful.

\section{LORA-BR and critical independent set problem}

Let $Q$ be an arbitrary graph. For a set $X \subseteq V(Q)$, let $N(X)=\cup_{x \in X}\{y \in V(Q)$ : $x y \in E(Q)\}$. Let $p, q$ be a pair of functions from $V(Q)$ to the set of non-negative reals. In the critical independent set problem (CISP) we seek $A$ maximizing

$$
\left\{\sum_{a \in A} p(a)-\sum_{c \in N(A)} q(c): A \text { is an independent vertex set in } Q\right\} .
$$

Clearly, CISP is $N P$-hard as the maximum weight independent set problem on arbitrary graphs is CISP with $q(u)=0$ for each $u \in V(Q)$. Ageev [1] proved that CISP is polynomial time solvable if $p(u)=q(u)$ for each $u \in V(Q)$. This generalized the corresponding result of Zhang [19] for $p(u)=q(u)=1$ for each $u \in V(Q)$. We will show that CISP can be solved in polynomial time on bipartite graphs for arbitrary functions $p$ and $q$.

Theorem 10. CISP on a bipartite graph $G=\left(V_{1}, V_{2} ; E\right), V=V_{1} \cup V_{2}$, can be solved in time $\mathrm{O}\left(n_{1}^{2} \sqrt{m}+n_{1} m+n\right)$, where $n_{1}=\left|V_{1}\right|, n=|V|$ and $m=|E|$.

Proof. Observe that LORA-BR with fixed lists $L_{1}=V_{1}, L_{2}=V_{2}$ may be reformulated as follows: given a bipartite graph $G=\left(V_{1}, V_{2}, E\right)$ and three weights $w_{i}(v), i=1,2,3$, for each vertex $v \in V$, we color every vertex of $G$ in one of the colors 1, 2, 3 such that if a vertex is colored 1 , then all its neighbors must be colored 3 . Assigning a color $i$ to a vertex $v$ contributes weight $w_{i}(v)$ to the total weight of the coloring. We seek a coloring of maximum total weight.

Observe that if $w_{1}(u)<w_{2}(u)$ for some $u \in V$, then there is an optimal coloring for which $u$ is not colored 1. Thus, we may set $w_{1}(u):=w_{2}(u)$ and keep a record, say $(u, 1,2)$, that indicates that if, in an optimal coloring that we found $u$ is colored 1 , we recolor it 2 . Similar arguments allow us to assume that $w_{1}(u) \geqslant w_{2}(u) \geqslant w_{3}(u)$ for each $u \in V$.

Consider an optimal coloring, in which $A$ is the set of vertices assigned color 1 . Then $A$ is independent, all vertices of $N(A)$ must have color 3 and all vertices of $B=V(G)-A-N(A)$ may have color 2 . The total weight of the coloring is

$$
\sum_{a \in A} w_{1}(a)+\sum_{c \in N(A)} w_{3}(c)+\sum_{b \in B} w_{2}(b)=\sum_{d \in V} w_{2}(d)-\sum_{c \in N(A)} w_{2,3}(c)+\sum_{a \in A} w_{1,2}(a)
$$

where $w_{2,3}(c)=w_{2}(c)-w_{3}(c), w_{1,2}(a)=w_{1}(a)-w_{2}(a)$.

Choose weight functions $w_{1}, w_{2}, w_{3}$ as follows: $w_{1}(u)=p(u)+q(u), w_{2}(u)=q(u), w_{3}(u)=0$ for each $u \in V(G)$. Since $\sum_{d \in V} w_{2}(d)$ is a constant, we observe that CISP on $G$ (and functions $p$ and $q$ ) can be reduced to LORA-BR with fixed $L_{1}=V_{1}, L_{2}=V_{2}$. It remains to apply Theorem 4 . 


\section{Acknowledgements}

We would like to thank Graham Brightwell, David Cohen and Martin Green for valuable discussions on the topic of the paper. Research of the first three authors was partially supported by the Leverhulme Trust. Research of Gutin and Rafiey was supported in part by the IST Programme of the European Community, under the PASCAL Network of Excellence, IST-2002-506778.

\section{References}

[1] A.A. Ageev, On finding critical independent and vertex sets, SIAM J. Discrete Math. 7 (1994) 293-295.

[2] R.K. Ahuja, J.B. Orlin, C. Stein, R.E. Tarjan, Improved algorithms for bipartite network flows, SIAM J. Comput. 23 (1994) 906-933.

[3] A.S. Asratian, T.M.J. Denley, R. Haggkvist, Bipartite Graphs and Their Applications, Cambridge University Press, Cambridge, 1998.

[4] L. Barros, The optimisation of repair decisions using life-cycle cost parameters, IMA J. Management Math. 9 (1988) $403-413$.

[5] L. Barros, Private communications with M. Tso, 2001.

[6] L. Barros, M. Riley, A combinatorial approach to level of repair analysis, European J. Oper. Res. 129 (2001) $242-251$.

[7] G. Brightwell, Private communications with G. Gutin, January, 2005.

[8] T.H. Cormen, C.E. Leiserson, R.L. Rivest, Introduction to Algorithms, MIT Press, Cambridge, MA, 1990.

[9] G. Cornuejols, G.L. Nemhauser, L.A. Wolsey, in: R.L. Francis, P.B. Mirchandani (Eds.), The uncapacitated facility location problem, Discrete Location Theory, Wiley, New York, 1990 (Chapter 3).

[10] J.W. Crabtree, B.C. Sandel, Army level of repair analysis (LORA), Logistics Spectrum (1989) 27-31.

[11] T. Feder, P. Hell, J. Huang, List homomorphisms and circular arc graphs, Combinatorica 19 (1999) 487-505.

[12] G. Frahling, U. Faigle, Combinatorial algorithm for weighted stable sets in bipartite graphs, Discrete Appl. Math., to appear.

[13] M.R. Garey, D.S. Johnson, Computers and Intractability, Freeman, San Francisco, 1979.

[14] B. Goldengorin, Requirements of Standards: Optimization Models and Algorithms, ROR Company, Hoogezand, The Netherlands, 1995.

[15] P. Hell, J. Nesetril, On the complexity of $H$-coloring, J. Combin. Theory B 48 (1990) 92-110.

[16] P. Hell, J. Nesetril, Graphs and Homomorphisms, Oxford University Press, Oxford, 2004.

[17] D. Hochbaum, Provisioning, shared fixed costs, maximum closure, and implications on algorithmic methods today, Management Sci. 50 (2004) 709-723.

[18] D. West, Introduction to Graph Theory, Prentice-Hall, Upper Saddle River, NJ, 1996.

[19] C.-Q. Zhang, Finding critical independent sets and critical vertex subsets are polynomial problems, SIAM J. Discrete Math. 3 (1990) $431-438$. 